\title{
The eccentric connectivity index of polycyclic aromatic hydrocarbons (PAHs)
}

\author{
Mehdi Alaeiyana $^{\mathrm{a}}$ |Chidambaram Natarajanb |G. Sathiamoorthyc ${ }^{\mathrm{b}}$ |Mohammad Reza Farahania,*
}

aDepartment of Mathematics, Iran University of Science and Technology (IUST) Narmak, 16844, Tehran, Iran

bepartment of Mathematics, Srinivasa Ramanujan Centre, SASTRA Deemed University, Kumbakonam-612 001, India

cDepartment of Mathematics, School of Arts, Science and Humanities, SASTRA Deemed University, Thanjavur-613 401, Tamil Nadu, India

\section{*Corresponding Author: \\ Mohammad Reza Farahani \\ Email: alaeiyan@iust.ac.ir \\ Tel.: +98 (9192478265)}

Mathematical chemistry is the area of research engaged in new application of Mathematics in Chemistry. Major areas of research in mathematical chemistry include chemical graph theory. Chemical graph theory applies graph theory to mathematical modeling of chemical phenomena. If $G=(V(G), E(G))$ is a connected graph, where $V(G)$ is a non-empty set of vertices and $E(G)$ is a set of edges, then the eccentric connectivity index of $G$ (denoted by $\xi(G)$ ) was defined as $\zeta(G)=\sum_{v \in V(G)} d_{v} \varepsilon(v)$, where $d_{v}$ is the degree of a vertex $v$ and $\varepsilon(v)$ is its eccentricity. In this study, we investigated the eccentric connectivity index of polycyclic aromatic hydrocarbons (PAHs).

\section{KEYWORDS}

Connected Molecular Graph; eccentric connectivity index; distance; polycyclic aromatic hydrocarbons (PAHs).

\section{Introduction}

Let $G=(V, E)$ be a simple connected graph with vertex set $V(G)$ and edge set $E(G)$. A molecular graph is a simple connected graph such that its vertices correspond to the atoms and the edges to the bonds. The degree of a vertex/atom $v$ in $G$ is the number of edges incident to it and is denoted by $d v$. The distance between the vertices $u$ and $v$, denoted by $d(u, v)$, is the length of the shortest path joining them. The eccentricity $\varepsilon(v)$ of a vertex $v$ is the distance to a vertex farthest from $v$, that is

$$
\varepsilon(v)=\operatorname{Max}\{d(u, v) \mid \forall u \in V(G)\}
$$

The radius $R(G)$ of a graph $G$ is the minimum eccentricity of the vertices and the diameter $D(G)$ of $G$ is the maximum eccentricity.

$$
\begin{gathered}
D(G)=\operatorname{Max}_{v \in V(G)}\{d(u, v) / \forall u \in V(G)\} \\
R(G)=\operatorname{Min}_{v \in V(G)}\{\operatorname{Max}\{d(u, v) \mid \forall u \in V(G)\}\}
\end{gathered}
$$

An eccentric vertex of a vertex $v$ is a vertex farthest away from $v$. An eccentric path of a vertex $v$ denoted by $P(v)$ is a path of length $e(v)$ joining $v$ and its eccentric vertex. There may exist more than one eccentric path for a given vertex.

A topological index of a graph is a number related to a graph which is invariant under graph automorphisms and is a numeric quantity from the structural graph of a molecule.

The eccentric connectivity index $\zeta(G)$ of a graph $G$ is defined by $V$. Sharma, R. Goswami, A.K. Madan, in 1997 [1], as

$$
\zeta(G)=\sum_{v \in V(G)}\left[d_{v} \times e c c(v)\right]
$$

Recently, we know the eccentric connectivity polynomial of a graph $G$ as

$$
\operatorname{ECP}(G, x)=\sum_{v \in(G)} d_{v} x^{\varepsilon(v)}
$$

Obviously, the eccentric connectivity index is the first derivative of $\operatorname{ECP}(G, x)$ evaluated at $x=1$. Also, the total eccentricity index of $G$ is defined as

$$
\Theta(G)=\sum_{v \in(G)} \varepsilon(v)
$$


M.J. Morgan et al. [2] investigated the eccentric connectivity index of trees. For further study and more details see references [3-6].

In this paper, we investigate the eccentric connectivity index of a family of hydrocarbon molecules, which are called Polycyclic Aromatic Hydrocarbons (PAHs).

\section{Results and discussion}

Mathematical chemistry is the area of research engaged in new application of mathematics in chemistry. Major areas of research in mathematical chemistry include chemical graph theory. Chemical graph theory applies graph theory to mathematical modeling of chemical phenomena.

Polycyclic aromatic hydrocarbons (PAHs) are a group of over 200 different chemicals formed when wood, coal, oil, gasoline or other organic materials are burned. They can also be formed in food when fish or meats are charbroiled. "Polycyclic aromatic hydrocarbon" is just a mouthful for an organic molecule in space-not just any organic molecule, but one with a structure sturdy enough to survive the harsh environment between stars. Usually called "PAHs," this class of molecule is almost certainly the culprit responsible for the unidentified infrared emission (UIR) bands discovered in the early 1970s. The UIR bands are seen in many different kinds of objects from starburst galaxies to comets, so understanding their origin is very important. Because the UIR bands are so common, the PAHs which produce them must account for a sizeable fraction of all interstellar carbon in our galaxy and others. Estimates run as high as $15 \%$ or more.

Polycyclic Aromatic Hydrocarbons (PAHs) consist several copies of benzene $\mathrm{C} 6$ on circumference and is ubiquitous combustion products. They have been implicated as carcinogens and play a role in graphitization of organic materials [7]. In addition, they are of interest as molecular analogues of graphite [8] as candidates for interstellar species [9] and as building blocks of functional materials for device applications [8-10]. Synthetic routes to PAHs are available [11] and a detailed knowledge of all these features would therefore be necessary for the tuning of molecular properties towards specific applications (see the references [7-12]).

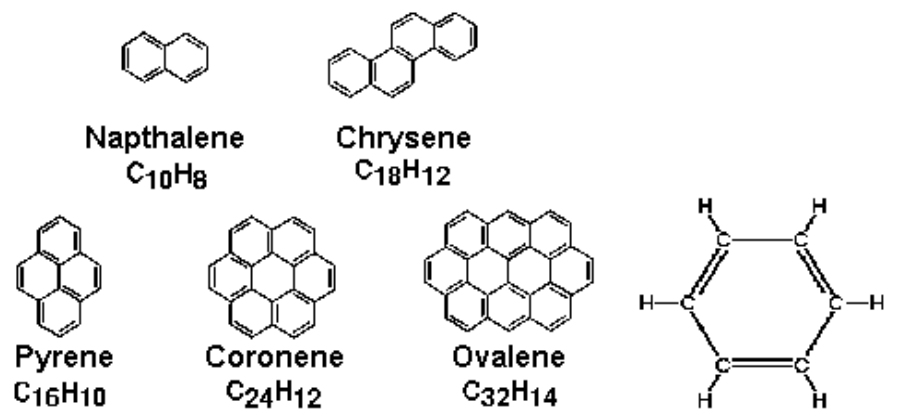

FIGURE 1 [20] Some example of polycyclic aromatic hydrocarbon $P A H_{s}$

The PAHs can be thought as small pieces of graphene sheets with the free valences of the dangling bonds saturated by $H$. Vice versa, a graphene sheet can be interpreted as an infinite PAH molecule. Successful utilization of PAH molecules in modeling graphite surfaces has been reported earlier [13-34] and references therein.
The first famous members of this hydrocarbon family (PAH family) are denoted and shown as follows (See Figures 1 and 2):

- $P A H_{1}$ be the Benzene with six carbon (C) and six hydrogen $(\mathrm{H})$ atoms,

- $\mathrm{PAH}_{2}$ be the Coronene with 24 carbon and twelve hydrogen atoms,

- $\mathrm{PAH}_{3}$ be the Circumcoronene with 54 carbon and eighteen hydrogen atoms. 


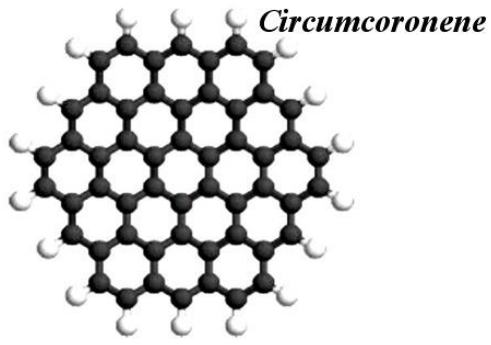

FIGURE 2 The first three graphs of polycyclic aromatic hydrocarbon $P A H_{n .}$. [26]

The general representation of polycyclic aromatic hydrocarbons (we denote $P A H_{n}$ ) is shown in Figure 3. One can see that the hydrocarbon $P A H_{n}$ has $6 n^{2}$ carbon (C) and $6 n$ hydrogen $(\mathrm{H})$ atoms.

Also, from Figure 3, it is easy to see that the maximum number of $\varepsilon_{v}$ is for hydrogen $(\mathrm{H})$ atoms. In other words,

$$
\begin{gathered}
D\left(P A H_{n}\right)=\operatorname{Max}_{v \in V(P A H n)}\left\{d(u, v) / \forall u \in V\left(P A H_{n}\right)\right\}=\varepsilon( \\
H)+1
\end{gathered}
$$

Now, from the general form of the polycyclic aromatic hydrocarbon $P A H_{n}$ and by using the ring-cut Method and we can achieve to our main results of this paper. In ring-cut method, all vertices of $G$ dividing into some partitions and, we insert some vertices of $G$ in a common ring-cut, such that these vertices have similar mathematical properties.

In other words, for computing the eccentric connectivity index $\zeta(G)$ of a graph $G$, all vertices in a common ring-cut are an equivalence eccentric.

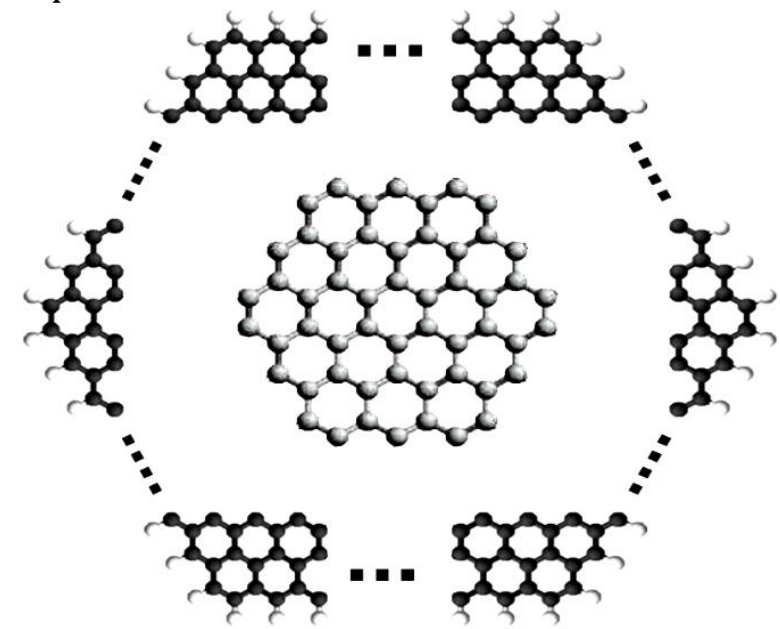

FIGURE 3 The general representation of polycyclic aromatic hydrocarbon $P A H_{n}$ for all integer number $n$ [22]
Now, by reference to the eccentric connectivity index $\zeta\left(H_{k}\right)$ of Circumcoronene series of Benzenoid $H_{k}$ [31-34] and figures therein (see Figure 3), we can compute the eccentric connectivity index $\zeta\left(\mathrm{PAH}_{n}\right)$ easily as: At first, we denote all vertices as degree three (carbon (C) atoms) of polycyclic aromatic hydrocarbon $P A H_{n}$ by $\beta$ and $\gamma$ (similar to Figure 4) and all vertices as degree two (hydrogen $(\mathrm{H})$ atoms) by $\alpha$. Alternatively, the vertex/atom set and edge/bond set of $P A H_{n}$ are equal to:

$$
\begin{gathered}
V\left(P A H_{n}\right)=\left\{\alpha_{z, l}, \beta_{z, l}^{i}, \gamma_{z, j}^{i} \mid I=1, \ldots, k, j \in \mathbb{Z}_{i} \&\right. \\
\left.l \in \mathbb{Z}_{i-1} \& z \in \mathbb{Z}_{6}\right\} \& \\
E\left(P A H_{n}\right)=\{ \\
\alpha_{z, j} \gamma_{z, j}^{k}, \beta_{z, j}^{i} \gamma_{z, j}^{i}, \beta_{z, j}^{i} \gamma_{z, j+1}^{i}, \beta_{z, j}^{i} \gamma_{z, j}^{i-1} \& \gamma_{z, i}^{i} \gamma_{z+1,1}^{i} \\
\left.\mid i \in \mathbb{Z}_{k} \& j \in \mathbb{Z}_{i} \& z \in \mathbb{Z}_{6}\right\}
\end{gathered}
$$

where $\mathbb{Z}_{i}=\{1,2, \ldots, i\}$ is the cycle finite group of order $i$.

So, we divide all vertices in some partitions "ring-cuts", such that a $i^{\text {th }}$ ring-cut consists of vertices $\beta_{z, j}^{i} \gamma_{z, j}^{i}\left(\forall i=1, . ., k ; z \in \mathbb{Z}_{6, j} \in \mathbb{Z}_{i}\right)$ and the size of this ring-cut is equal to $6 i+6(i-1)$. Also, one common property of members of a ringcut is their farthest vertices. And another properties of them is $d\left(\gamma_{z, j}^{i}, \gamma_{z, j}^{k}\right)=d\left(\beta_{z, l}^{i}, \beta_{z, l}^{k}\right)$ $=2(k-i)$, in other words the distance of these vertices are equal to two times of difference between order of their ring-cuts. By attention to ring cuts of polycyclic aromatic hydrocarbon $P A H_{n}$ and using above mentions and results from [29], we have following formula:

- For all vertices $\beta_{z, j}^{i}$ of $P A H_{k}\left(\forall i=1, . ., k ; z \in \mathbb{Z}_{6,}\right.$ $\left.j \in \mathbb{Z}_{i-1}\right)$ $\varepsilon\left(\beta_{z, j}^{i}\right)=\underbrace{d\left(\beta_{z, j}^{i}, \beta_{z+3, j}^{i}\right)}_{4 i-3}+\underbrace{d\left(\beta_{z+3, j}^{i}, \gamma_{z+3, j}^{k}\right)}_{2(k-i)+1}+\underbrace{d\left(\gamma_{z+3, j}^{k}, \alpha_{z+3, j}\right)}_{1}$ $=2 k+2 i-1$

- For all vertices $\gamma_{z, j}^{i}$ of $P A H_{n}\left(\forall i=1, . ., k ; z \in \mathbb{Z}_{6,}\right.$ $\left.j \in \mathbb{Z}_{i}\right)$

$$
\varepsilon\left(\gamma_{z, j}^{i}\right)=\underbrace{d\left(\gamma_{z, j}^{i}, \gamma_{z+3, j}^{i}\right)}_{4 i-1}+\underbrace{d\left(\gamma_{z+3, j}^{i}, \gamma_{z+3, j}^{k}\right)}_{2(k-i)}+\underbrace{d\left(\gamma_{z+3, j}^{k}, \alpha_{z+3, j}\right)}_{1}
$$


$=2(k+i)$

- For all vertices $\alpha_{z, j}$ (hydrogen (H) atoms) of $P A H_{n}(\forall j=1, . ., k ; z=1, . ., 6)$

$\varepsilon\left(\alpha_{z, j}\right)=\underbrace{d\left(\alpha_{z, j}, \gamma_{z, j}^{k}\right)}_{1}+\underbrace{d\left(\gamma_{z, j}^{k}, \gamma_{z^{\prime}, j^{\prime}}^{k}\right)}_{4 k-1}+\underbrace{d\left(\gamma_{z^{\prime}, j^{\prime}}^{k}, \alpha_{z^{\prime}, j^{\prime}}\right)}_{1}$

$=4 k+1$

- The diameter number of $P A H_{n}$ is equal to $D\left(P A H_{n}\right)=\varepsilon(H)=4 k+1$.

- The radius number of $H_{k}$ is equal to $R\left(H_{k}\right)=2 k+1$.

Now, we are in a position to state and prove our main results of this paper. Therefore, the eccentric connectivity index $\zeta$ of polycyclic aromatic hydrocarbon $P A H_{n}$ is equal to:

$\xi\left(P A H_{n}\right)=\sum_{v \in V\left(P A H_{n}\right)} d_{v} \times \varepsilon(v)$

$=\sum_{\alpha_{z, j}} d_{\alpha_{z, j}} \varepsilon\left(\alpha_{z, j}\right)+\sum_{i=2}^{n} \sum_{\beta_{z, j}^{i}} d_{\beta_{z, j}^{i}} \varepsilon\left(\beta_{z, j}^{i}\right)+\sum_{i=1}^{n} \sum_{\gamma_{z, j}^{i}} d_{\gamma_{z, j}^{i}} \varepsilon\left(\gamma_{z, j}^{i}\right)$

$=\sum_{i=1}^{n}\left(\sum_{z=1}^{6} d_{\alpha_{z,}} \varepsilon\left(\alpha_{z, i}\right)+\sum_{j=1}^{i-1} \sum_{z=1}^{6} d_{\beta_{z, j}^{i}} \varepsilon\left(\beta_{z, j}^{i}\right)+\sum_{j=1}^{i} \sum_{z=1}^{6} d_{\gamma_{z, j}} \varepsilon\left(\gamma_{z, j}^{i}\right)\right)$

$\sum_{z=1}^{6}\left(\sum_{j=1}^{n} d_{\alpha_{z, j}} \varepsilon\left(\alpha_{z, j}\right)+\sum_{i=2}^{n} \sum_{j=1}^{i-1} d_{\beta_{z, j}^{i}} \varepsilon\left(\beta_{z, j}^{i}\right)+\sum_{i=1}^{n} \sum_{j=1}^{i} d_{\gamma_{z, j}^{i}} \varepsilon\left(\gamma_{z, j}^{i}\right)\right)$

$$
=\sum_{z=1}^{6}\left(d_{H} \sum_{j=1}^{n} \varepsilon\left(\alpha_{z, j}\right)+d_{C} \sum_{i=2}^{n} \sum_{j=1}^{i-1} \varepsilon\left(\beta_{z, j}^{i}\right)+d_{C} \sum_{i=1}^{n} \sum_{j=1}^{i} \varepsilon\left(\gamma_{z, j}^{i}\right)\right)
$$

$=6\left(1 \sum_{j=1}^{n} \varepsilon\left(\alpha_{z, j}\right)+3 \sum_{i=2}^{n} \sum_{j=1}^{i-1} \varepsilon\left(\beta_{z, j}^{i}\right)+3 \sum_{i=1}^{n} \sum_{j=1}^{i} \varepsilon\left(\gamma_{z, j}^{i}\right)\right)$

$=6\left(\sum_{j=1}^{n}(4 n+1)+3 \sum_{i=2}^{n} \sum_{j=1}^{i-1}(2 n+2 i-1)+3 \sum_{i=1}^{n} \sum_{j=1}^{i} 2(n+i)\right)$

$=6\left(\sum_{i=1}^{n}(4 n+1)+3 \sum_{i=2}^{n}(i-1)(2 n+2 i-1)+3 \sum_{i=1}^{n} 2 i(n+i)\right)$

$=6\left(\sum_{i=1}^{n}(4 n+1)+3 \sum_{i=1}^{n}(i-1)(2 n+2 i-1)+3 \sum_{i=1}^{n} 2 i(n+i)\right)$

$=6\left(\left(4 n^{2}+n\right)+3 \sum_{i=1}^{n}\left(4 i^{2}+(4 n-3) i-2 n+1\right)\right)$

$=6\left(\left(4 n^{2}+n\right)+3\left(\frac{10 n^{3}}{3}+\frac{n^{2}}{2}+\frac{n}{6}\right)\right)$

$=6\left(10 n^{3}+11 / 2 n^{2}+3 / 2 n\right)$

$=3 n\left(20 n^{2}+11 n+3\right)$.

Thus the eccentric connectivity index of polycyclic aromatic hydrocarbon $P A H_{n}$ is $\xi\left(P A H_{n}\right)=60 n^{3}+33 n^{2}+9 n$.

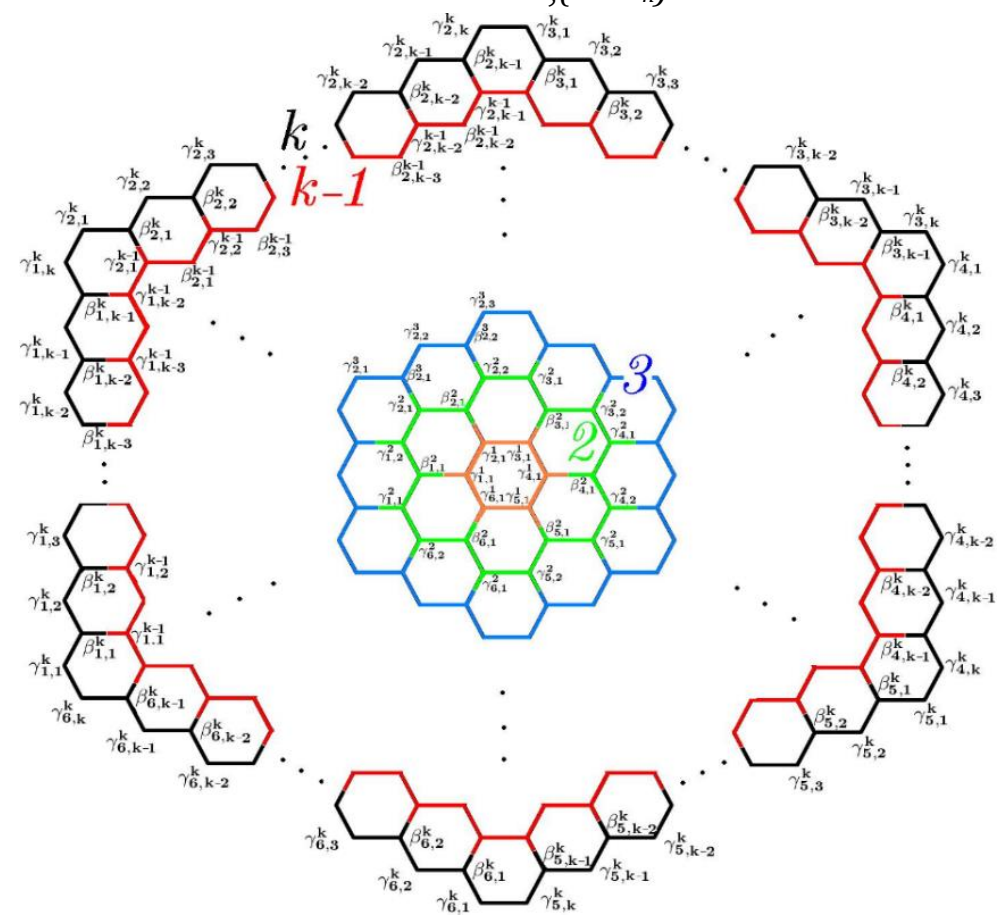

FIGURE 4 The general representation of ring-cut method of circumcoronene series of benzenoid $H_{k}$, for all integer number $k \geq 2$ [31-34] 
Example 1. Consider Benzene $\mathrm{PAH}_{1}$ (Figure 1 ), by using the above results, the eccentric connectivity index of Benzene is equal to $\xi\left(P A H_{n}\right)=102$.

Example 2. Consider Coronene $\mathrm{PAH}_{2}$ (Figure 1), by using the above results, one can see that the eccentric connectivity index of the second member of polycyclic aromatic hydrocarbon is equal to $\xi\left(P A H_{n}\right)=630$.

\section{Conclusion}

In graph theory, we have many invariant polynomials for a graph $G$. In this research, we focus on the structure of Polycyclic Aromatic Hydrocarbons (PAHs). We have computed the eccentric connectivity index of this hydrocarbon molecule.

\section{Acknowledgments}

The authors would like to thank the referee for his/her helpful suggestions and comments.

\section{Orcid:}

Mohammad Reza Farahani:

https://orcid.org/0000-0003-2969-4280

\section{References}

[1] V. Sharma, R. Goswami, A.K. Madan, J. Chem. Inf. Model., 1997, 37, 273-282.

[2] M.J. Morgan, S. Mukwembi, H.C. Swart, Discrete Mathematics, 2011, 311, 1229-1234.

[3] A.R. Ashrafi, M. Ghorbani, M. Hemmasi, Dig. J. Nanomater. Bios., 2009, 4, 483-486.

[4] S. Alikhani, M.A. Iranmanesh, Dig. J. Nanomater. Bios., 2011, 6, 253-257.

[5] I. Gutman, O.E. Polansky, Springer-Verlag, New York, 1998.

[6] M.A. Johnson, G.M. Maggiora, Wiley Interscience, New York, 1990.

[7] U.E. Wiersum, L.W. Jenneskens, Amsterdam., The Netherlands, 1997, 143194.

[8] A.J. Berresheim, M. Muller, K. Müllen, Polyphenylene nanostructures. Chem. Rev., 1999, 99, 1747-1785.
[9] C.W. Bauschlicher, Jr, E.L.O. Bakes, Chem. Phys., 2000, 262, 285-291.

[10] A.M. Craats, J.M. Warman, K. Müllen, Y. Geerts, J. D. Brand, Adv. Mater., 1998, 10, 3638.

[11] F. Morgenroth, C. Kübel, M. Müller, U.M. Wiesler, A.J. Berresheim, M. Wagner, K. Müllen, Carbon, 1998, 36, 833- 837.

[12] F. Dotz, J.D. Brand, S. Ito, L. Ghergel, K. Müllen, J. Am. Chem. Soc., 2000, 122, 77077717

[13] K. Yoshimura, L. Przybilla, S. Ito, J.D. Brand, M. Wehmeir, H.J. Rder, K. Müllen, Macromol. Chem. Phys., 2001, 202, 215-222.

[14] S.E. Stein, R.L. Brown, J. Am. Chem. Soc., 1987, 109, 3721-3729.

[15] F. Dietz, N. Tyutyulkov, G. Madjarova, K. Müllen, J. Phys. Chem. B, 2000, 104, 17461761.

[16] J.B. Liu, X.F. Pan, L. Yu, D. Li, Discrete Appl. Math., 200, 2016, 95-107.

[17] J.B. Liu, X.F. Pan, Appl. Math. Comput., 2016, 291, 84-88.

[18] J.B. Liu, W.R. Wang, Y.M. Zhang, X.F. Pan, Discrete Appl. Math., 2016, 203, 217-225.

[19] J.B. Liu, X.F. Pan, F.T. Hu, F.F. Hu, Appl. Math. Comput., 2015, 253, 205-214.

[20] J.B. Liu, Ch., Wang, Sh. Wang, B. Malys. Math. Sci. So., 2019, 42, 67-78.

[21] J.B. Liu, X.-F. Pan, Appl. Math. Comput., 2016, 291, 84-88.

[22] J.B. Liu, J. Zhao, Zh. Zhu, Int J Quantum Chem., 2019, e25971.

[23] A. Soncini, E. Steiner, P.W. Fowler, R.W.A. Havenith, L.W. Jenneskens, Chem. Eur.J., 2003, 9, 2974-2981.

[24] K. Jug, T. Bredow, J. Comput. Chem., 2004, 25, 1551-1567.

[25] L. Guerrini, J.V. Garcia-Ramos, C. Domingo, S. Sanchez-Cortes, Anal. Chem., 2009, 81, 953-960.

[26] Webpage: G.C. Sloan Polycyclic aromatic hydrocarbons PAHs. http://isc.astro.cornell.edu/ sloan/research /pah_info.html 
[27] J.H. Pacheco-Sanchez, G.A. Mansoori, Revista. Mexicana. de Fisica., 2013, 59, 584593.

[28] M.R. Farahani, Adv. Mater. Corrosion, 2013, 1, 65-69.

[29] M.R. Farahani, Letters of Chemistry, Physics and Astronomy., 2014, 13, 1-10.

[30] M.R. Farahani, W. Gao, Journal of Chemical and Pharmaceutical Research, 2015, 7, 535539.

[31] M.R. Farahani, M.R.R. Kanna, Journal of Chemical and Pharmaceutical Research, 2015, 7, 253-257.

[32] L. Yan, Y. Li, M.R. Farahani, M. Imran, M.R.R. Kanna, J. Comput. Theor. Nanosci., 2016, 13, 8874-8878.

[33] M.R. Farahani, World Appl. Sci. J., 2013, 21, 1260-1265.

[34] M.R. Farahani, Annals of West University of Timisoara-Mathematics and Computer Science. 2013, 51, 29-37.
[35] M.R. Farahani, Journal of Chemica. Acta., 2013, 2, 26-31.

[36] M.R. Farahani, Journal of Chemica. Acta., 2013, 2, 22-25.

How to cite this article: Mehdi Alaeiyan, Chidambaram Natarajan, G. Sathiamoorthy, Mohammad Reza Farahani*. The eccentric connectivity index of polycyclic aromatic hydrocarbons (PAHs). Eurasian Chemical Communications, 2020, 2(6), 646-651. Link: http://www.echemcom.com/article_10478 $0 . h$ tml 